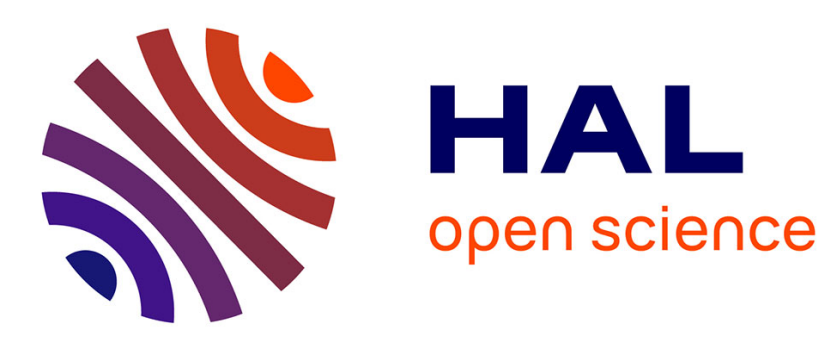

\title{
Using singular value decomposition of component eigenmodes for interface reduction
}

Hadrien Tournaire, Franck Renaud, Jean-Luc Dion

\section{To cite this version:}

Hadrien Tournaire, Franck Renaud, Jean-Luc Dion. Using singular value decomposition of component eigenmodes for interface reduction. Journal of Sound and Vibration, 2018, 414, pp.1 - 14. 10.1016/j.jsv.2017.05.029 . hal-01644371

\section{HAL Id: hal-01644371 \\ https://hal.science/hal-01644371}

Submitted on 22 Nov 2017

HAL is a multi-disciplinary open access archive for the deposit and dissemination of scientific research documents, whether they are published or not. The documents may come from teaching and research institutions in France or abroad, or from public or private research centers.
L'archive ouverte pluridisciplinaire HAL, est destinée au dépôt et à la diffusion de documents scientifiques de niveau recherche, publiés ou non, émanant des établissements d'enseignement et de recherche français ou étrangers, des laboratoires publics ou privés. 


\title{
Using singular value decomposition of component eigenmodes for interface reduction
}

\author{
Hadrien Tournaire ${ }^{\mathrm{a}}$, Franck Renaud ${ }^{\mathrm{b}}$, Jean Luc Dion ${ }^{\mathrm{b}}$ \\ ${ }^{a}$ IRT SytemX, 8 Avenue de la Vauve, 91120 Palaiseau \\ ${ }^{b}$ SUPMECA, QUARTZ EA7393, 3 rue Fernand Hainaut, 93407 Saint Ouen
}

\begin{abstract}
The aim of this paper is to describe the development of a reduced order model for modal analysis in a design context. The design process of most industrial systems is based on the re-utilization of certain components. Here, we propose a reduction method involving component eigenmodes to recover the modal behaviour of an assembled structure. The contribution of this work is that it uses component eigenmodes to build an interface reduction basis. Lastly, the reduction methodology proposed is compared to the Craig and Bampton method by applying it to two case studies of which one is an industrial model of an open rotor blade.
\end{abstract}

Keywords: Modal analysis, Free component eigenmodes, Interface reduction

\section{Introduction}

The mechanical design of a system involves numerous investigations, including in particular the validation of its dynamical behaviour over its operating frequency range. This type of analysis can be performed numerically using the finite element method. However, in this context, the accuracy and level of detail required involve models whose significant sizes lead to time-consuming simulations. Moreover, the optimization process of such a system may demand a large number of validation computations that considerably slow down the design process. In this paper, the industrial case study considered is of modest size by current standards but subject to tens of redesign iterations. It is managed using a basic laptop.

Nowadays, reduction methods are used industrially to solve large and complex structural dynamics problems and reduce simulation time. Recent reviews of these methods were carried out in $[1,2]$. These methods allow evaluating the behaviour of models (sometimes in real-time [3]) incorporating several million degrees of freedom (DoF), see for example $[4,5,6]$. The dynamic substructuring technique (DS), first initiated by Hurty in the early 1960s, was of paramount importance in this progress. Indeed, the basic idea of this technique is to consider a large model as an assembly of smaller models that are easier to handle. Two main families can be identified [7] among these DS-based methods, namely Direct Coupling (DC) and Component Mode Synthesis (CMS). Whereas direct coupling deals with the enforcement of the Dirichlet and/or Neumann conditions on the contact interfaces in the nodal space, the CMS method imposes these conditions in the reduced space through the choice of the reduction vectors [8]. Indeed, CMS methods often consider the reduction of independent component finite element models whose interactions are described with modes.

The coupling condition associated with dynamic substructuring methods (primal, dual or hybrid) also has a significant impact on the performance of the reduced order model. Primal coupling focuses on maintaining conformity with the Dirichlet boundary conditions between the contacting components, to ensure the

Email addresses: hadrien.tournaire@irt-systemx.fr (Hadrien Tournaire), franck.renaud@supmeca.fr (Franck Renaud), jean-luc.dion@supmeca.fr (Jean Luc Dion) 
accurate description of the displacement fields of the structure assembled. The dual coupling formulation is based on the Neumann condition so that the coupling condition is weakly enforced.

\section{Nomenclature}

$\mathbf{K}, \mathbf{M}, \mathbf{Z}$ stiffness matrix, mass matrix, dynamic stiffness matrix

$\boldsymbol{\Lambda}_{k}$ spectral matrix associated to structure $k$

$\mathbf{I}_{n}$ identity matrix of size $n \times n$

$\mathbf{u}, \mathbf{f}$ displacement, force vector

$\omega$ circular frequency

$\star_{i}$ internal DoF of $\star$

$\star_{b}$ boundary DoF of $\star$ $\boldsymbol{\phi}_{k}$ free eigenmode of the structure $\Sigma_{k}$

$\psi_{k}$ eigenmode of the structure $\Sigma_{k}$ where the $b$ DoF are fixed

$\star^{\top}$ transpose of $\star$

$\approx$ reduced variable $\star$

$n_{k}$ number of DoF associated with the structure $\Sigma_{k}$

$n_{\boldsymbol{\Phi}_{k}}$ number of vectors $\boldsymbol{\Phi}_{k}$

The advantages provided by dynamic substructuring methods to structural dynamics are numerous [7]. They allow evaluating the behaviour of a structure that is too large or too complex to be analysed as a whole [4]. Indeed, the matrix inversion and eigensolution algorithm lead to quite considerable computational costs, thus the beneficial aspect of breaking down a large problem into a set of small ones is immediate. Moreover, as substructuring involves independent computations on the components, the parallelism achieved is widely used in classical methods such as the Craig and Bampton, MacNeal, FETI (Finite Element Tearing and Interconnecting) [6] and AMLS (Automated Multi-Level Solver ) $[9,10]$ methods, as well as in more recent methods [4].

Another feature provided by substructuring is that the contribution of each component to the overall response of the structure assembled is identified. This investigation can be achieved using the free eigenmodes of components as reduction vectors. From the design point of view, this type of analysis provides access to data that can be used to guide the redesign of components independently. Certain dual CMS methods using component free eigenmodes can be cited, such as the Mac-Neal method [11] and, more recently, the dual Craig and Bampton method developed by Rixen [12] and [13, 14, 15]. Nevertheless these methods are based on maintaining continuous force at the contact interface. Such an approach may not be well-adapted when the displacement field is of primary importance. It is noteworthy that hybrid methods mixing both primal and dual techniques can provide a good trade-off between the description of the force and displacement fields [16].

Another great advantage provided by dynamic substructuring is linked to its capacity to enrich the model assembled with experimental measurements and to combine them with other models. It is thus possible to evolve from an initial model as the product design progresses, i.e. using experimental data from fabricated parts of the assembly and increasing its level of detail by combining it with other substructures. In other words, the assembled model of a system can be progressively enriched by taking into account the contribution of an increasing number of components.

In the present work, we seek a good description of the displacement field, justifying the use of reduction methods based on primal coupling. Martinez et al. [17] showed that primal coupling can be conjugated with the use of component eigenmodes as reduction vectors. While using free-free eigenmodes allows describing component behaviours, improvement vectors are essential to accurately recover the kinematics of the system assembled. Thus the major difficulty of this approach is that of choosing the improvement vectors that will be easy to compute and which correctly represent the potential interface motions. Also, their number should not be linked to the size of the contact interface $[18,19,4,20,14,21]$. 
To sum up, the aim of this work is to propose a reduction methodology with the following requirements:

- The reduction should provide a compact reduced order model: this goal is achieved through interface reduction.

- The reduction method must be oriented towards the modal analysis of the system assembled.

- The method's accuracy must be acceptable. This will be verified by comparing it with the Craig \& Bampton method.

This paper proposes a kinematic reduction methodology that relies on primal direct coupling (DC). The modal behaviour of the structure is recovered using a Ritz subspace spanned by component free eigenmodes and enrichment vectors. These enrichment vectors are obtained with different circular frequencies $\omega$. Classically, reduction methods like the Craig and Bampton method are limited by interface size. The central idea of the present work is to reduce the interface using the component free-free eigenmodes. The interface modes are evaluated using the singular value decomposition (SVD) of the component eigenmode interface restriction. This approach allows building a reduced order model using component free eigenmodes whose size and accuracy can be tuned by selecting SVD-interface modes. Indeed, when looking for the $N$ first natural modes, $N$ different interface vectors are obtained at most by limiting the $\mathrm{N}$ natural modes at the interface. $N$ does not depend on the number of interface DoFs; however, in Craig-like methods the reduction basis does. This is why the method proposed tries to retrieve these $N$ different interface vectors, regardless of the quality of the interface mesh. Two radically different types of situation may occur:

- Sometimes, as in the first case study, the interface mesh is coarse and the interface is large compared to the volume. Thus a high number of interface vectors are required compared to the number of interface DoFs. In this case, our method provides no advantages in comparison to Craig-like methods, but it gives a similar level of accuracy.

- Sometimes, as in the industrial case study (the second study described in this paper), the interface mesh is fine and the interface is small compared to the volume. Thus few interface vectors are required compared to the number of interface DoFs. This leads to a very small reduced order model compared to Craig-like models and, once again, to more or less the same accuracy. In this case the advantages of our method are obvious.

The reduction method proposed is well suited to the design context as it allows building updatable reduced order models. Indeed, the modification of certain components does not require the total reconstruction of the reduced order model.

This paper is organized as follows: the mechanical problem to be solved is first formalized in section 2 . Then, the primal component mode synthesis and the Craig and Bampton method are recalled in section 3. The method proposed is presented in section 4. The three key points of the proposed method are highlighted in the following order of priority: the assembly of the model using a primal direct approach, the reduction of the model using free component eigenmodes (section 4.1), and interface reduction (section 4.2). The generalisability of the method is first highlighted through its application to an academic case study with complex interface dynamic behaviour. Lastly, a comparison of the reduction method proposed with the Craig \& Bampton method is performed on the blade of an open rotor aircraft engine in section 5 .

\section{Definition of the mechanical problem}

In this section the mechanical problem to be reduced is introduced. First, the finite element modelling of the structural problem is presented in section 2.1, then the principle of the primal coupling is explained in section 2.2 . 


\subsection{Finite element modelling}

Let us consider a structure $\Sigma$ made of two components $\Sigma_{1}$ and $\Sigma_{2}$, involving $n_{1}$ and $n_{2}$ DoF, respectively. The two substructures are tied to each other through the contact interface denoted $\Gamma$, called transfer interface (Fig.1), to illustrate our purpose.

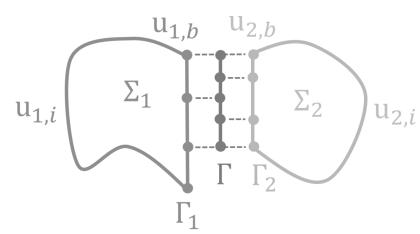

Figure 1: Example of two contacting finite element models with non-conforming meshes on their contacting surfaces $\Gamma_{1}$ and $\Gamma_{2}$.

This work deals only with the reduction of a linear conservative model. Thus the behaviour of the components considered is taken as purely linear elastic without any kind of dissipation. Considering a deformable body subject to this assumption, the finite element method and the projection into the Fourier domain allow obtaining a discrete model of its structural dynamics. Using $\mathbf{u}$ and $\mathbf{f}$ as the Fourier transform of the displacement and the external forces applied to the structure, the matrix equation of this linear model takes the form of the following well-known dynamic equation:

$$
\left(-\omega^{2} \mathbf{M}+\mathbf{K}\right) \mathbf{u}=\mathbf{Z}(\omega) \mathbf{u}=\mathbf{f}
$$

Although this article does not consider dissipative effects, certain works have been performed, notably by [22], to solve damped structures in a substructuring context. Strategies for the computation of eigenmodes taking dissipation into account are recalled in [23].

The finite element operators Eq.(1) of each component $\Sigma_{1}$ and $\Sigma_{2}$ can be partitioned and split into several sub-operators. The superscripts $b$ and $i$ refer to boundary (contacting) and interior DoFs, respectively. Using this notation, the finite element sub-operators are defined in the following manner:

$$
\begin{aligned}
& {\left[\begin{array}{ll}
\mathbf{Z}_{1, i i} & \mathbf{Z}_{1, i b} \\
\mathbf{Z}_{1, b i} & \mathbf{Z}_{1, b b}
\end{array}\right]\left\{\begin{array}{l}
\mathbf{u}_{1, i} \\
\mathbf{u}_{1, b}
\end{array}\right\}=\left\{\begin{array}{l}
\mathbf{f}_{1, i} \\
\mathbf{f}_{1, b}
\end{array}\right\}} \\
& {\left[\begin{array}{ll}
\mathbf{Z}_{2, i i} & \mathbf{Z}_{2, i b} \\
\mathbf{Z}_{2, b i} & \mathbf{Z}_{2, b b}
\end{array}\right]\left\{\begin{array}{l}
\mathbf{u}_{2, i} \\
\mathbf{u}_{2, b}
\end{array}\right\}=\left\{\begin{array}{l}
\mathbf{f}_{2, i} \\
\mathbf{f}_{2, b}
\end{array}\right\}}
\end{aligned}
$$

The physical contact interface is discretized twice for the model substructuring, by $\Gamma_{1}$ and $\Gamma_{2}$. Similarly, the force vectors $\mathbf{f}_{1, b}$ and $\mathbf{f}_{2, b}$ are used to represent the same force. Thus, these two vectors are the same when the interface meshes conform.

\subsection{Primal coupling}

The primal coupling condition relies on maintaining the displacement (Dirichlet) boundary condition on the interfaces in contact. In other words, the coupling strategy chosen has to ensure that the displacement fields on the contact interface are similar. Thus the displacement gap $\Delta \mathbf{u}^{\Gamma}$ is expected to be null for all the DoFs of the transfer interface $\Gamma$. The computation of the gap $\Delta \mathbf{u}^{\Gamma}$ requires the displacement vectors of components $\Sigma_{1}$ and $\Sigma_{2}$ on the set of transfer DoFs denoted $\mathbf{u}_{1, \Gamma}$ and $\mathbf{u}_{2, \Gamma}$, respectively:

$$
\Delta \mathbf{u}^{\Gamma}=\mathbf{u}_{1, \Gamma}-\mathbf{u}_{2, \Gamma}=\mathbf{0}
$$


Knowing the nodal displacement $\mathbf{u}_{1}$ and $\mathbf{u}_{2}$ of the component, it is possible to compute the displacement $\mathbf{u}_{1, \Gamma}$ and $\mathbf{u}_{2, \Gamma}$ of the interface $\Gamma$ thanks to the finite element interpolation function. In practice, this interpolation is performed using the interpolation matrix denoted $\left[\mathrm{L}_{\Sigma_{1}}^{\Gamma}\right]$ and $\left[\mathrm{L}_{\Sigma_{2}}^{\Gamma}\right]$ so that

$$
\mathbf{u}_{1, \Gamma}=\left[\mathrm{L}_{\Sigma_{1}}^{\Gamma}\right] \mathbf{u}_{1} \text { and } \mathbf{u}_{2, \Gamma}=\left[\mathrm{L}_{\Sigma_{2}}^{\Gamma}\right] \mathbf{u}_{2}
$$

In the case of conforming contacting meshes, the matrixes are boolean. The primal constraint Eq.(4) can be written as a matrix using the displacement vector $\mathbf{u}_{1}$ and $\mathbf{u}_{2}$ as follows

$$
\Delta \mathbf{u}^{\Gamma}=\mathbf{u}_{1, \Gamma}-\mathbf{u}_{2, \Gamma}=\left[\left[\mathrm{L}_{\Sigma_{1}}^{\Gamma}\right]-\left[\mathrm{L}_{\Sigma_{2}}^{\Gamma}\right]\right]\left\{\begin{array}{l}
\mathbf{u}_{1} \\
\mathbf{u}_{2}
\end{array}\right\}=\mathbf{0}
$$

For the sake of simplicity it is assumed in the next sections that the meshes of the contact interfaces are initially regularized (or basically conform) and that the DoF set $\mathbf{u}_{1, b}$ and $\mathbf{u}_{2, b}$ is sorted similarly in the models Eq.(2) and Eq.(3).

In the present work, the constraint Eq.(4) is enforced by searching the solution in a reduced subspace that exactly verifies Eq.(4).

\section{Interesting basics in primal component mode synthesis}

In this section the implementation of the primal coupling condition Eq.(4) in a component mode synthesis method is studied (section 3.1). Secondly, the Craig and Bampton method is recalled (section 3.2) as it is a widely-used and accurate method for primal component mode synthesis.

\subsection{Primal component mode synthesis}

The particularity of component mode synthesis is that the component is coupled using a reduction basis T. Generally, a reduced model using component mode synthesis based methods can be written as:

$$
\tilde{\mathbf{Z}} \tilde{\mathbf{u}}=\tilde{\mathbf{f}}
$$

Using $\mathbf{T}$ as the reduction basis for component mode synthesis, the reduced matrix and vectors of the assembled model are defined as:

$$
\tilde{\mathbf{Z}}=\mathbf{T}^{\top}\left[\begin{array}{cc}
\mathbf{Z}_{1} & \mathbf{0} \\
\mathbf{0} & \mathbf{Z}_{2}
\end{array}\right] \mathbf{T}, \mathbf{T} \tilde{\mathbf{u}}=\left\{\begin{array}{l}
\mathbf{u}_{1} \\
\mathbf{u}_{2}
\end{array}\right\}, \tilde{\mathbf{f}}=\mathbf{T}^{\top}\left\{\begin{array}{l}
\mathbf{f}_{1} \\
\mathbf{f}_{2}
\end{array}\right\}
$$

Where $\mathbf{Z}_{1}$ and $\mathbf{Z}_{2}$ are the component dynamic stiffnesses of the components $\Sigma_{1}$ and $\Sigma_{2}$, respectively. Therefore, the primal coupling condition Eq.(6) can be written using a set of reduced coordinates as:

$$
\Delta \mathbf{u}^{\Gamma}=\left[\left[\mathrm{L}_{\Sigma_{1}}^{\Gamma}\right]-\left[\mathrm{L}_{\Sigma_{2}}^{\Gamma}\right]\right] \mathbf{T} \tilde{\mathbf{u}}=\mathbf{0} \text { where } \tilde{\mathbf{u}} \neq \mathbf{0}
$$

The previous Eq.(8) can be simplified as follows:

$$
\Delta \mathbf{u}^{\Gamma}=\left(\left[\left[\mathrm{L}_{\Sigma_{1}}^{\Gamma}\right]-\left[\mathrm{L}_{\Sigma_{2}}^{\Gamma}\right]\right]\left[\begin{array}{l}
\mathbf{T}_{1} \\
\mathbf{T}_{2}
\end{array}\right]\right) \tilde{\mathbf{u}}=\left[\begin{array}{ll}
\mathbf{T}_{1, \Gamma} & -\mathbf{T}_{2, \Gamma}
\end{array}\right] \tilde{\mathbf{u}}=\mathbf{0}
$$

\subsection{Craig and Bampton method}

The Craig and Bampton method is a widely implemented component mode synthesis method based on a primal coupling approach. This method uses fixed-interface component eigenmodes $\boldsymbol{\psi}_{1}$ and $\boldsymbol{\psi}_{2}$ of both components $\Sigma_{1}$ and $\Sigma_{2}$ (thus $\boldsymbol{\psi}_{1, b}$ and $\boldsymbol{\psi}_{2, b}$ are null by definition). This provides the description of the internal behaviours of the components and the necessary displacement continuity common to primal component mode synthesis (see Eq.(9)) methods, so that: 


$$
\boldsymbol{\psi}_{1}=\left[\begin{array}{c}
\boldsymbol{\psi}_{1, i} \\
\mathbf{0}
\end{array}\right] \text { and } \boldsymbol{\psi}_{2}=\left[\begin{array}{c}
\boldsymbol{\psi}_{2, i} \\
\mathbf{0}
\end{array}\right]
$$

The interface displacements are described using a nodal displacement basis $\mathbf{T}_{b}$. In order to express the displacement continuity between the boundary and internal DoF, a Guyan condensation of the contacting component around the interface displacements $\mathbf{T}_{b}$ is performed:

$$
\boldsymbol{\Theta}_{1}=\left[\begin{array}{c}
-\mathbf{K}_{1, i i}^{-1} \mathbf{K}_{1, i b} \\
\mathbf{I}_{n_{b}}
\end{array}\right] \mathbf{T}_{b} \text { and } \boldsymbol{\Theta}_{2}=\left[\begin{array}{c}
-\mathbf{K}_{2, i i}^{-1} \mathbf{K}_{2, i b} \\
\mathbf{I}_{n_{b}}
\end{array}\right] \mathbf{T}_{b}
$$

The basis used to describe the interface displacements is nodal so that $\mathbf{T}_{b}$ corresponds to the identity $\mathbf{I}_{n_{b}}$ of size $n_{b} \times n_{b}$. Finally, the topology of the Craig and Bampton reduction basis $\mathbf{T}$ takes the following form:

$$
\mathbf{T}=\left[\begin{array}{ccc}
\boldsymbol{\Psi}_{1, i} & \mathbf{0} & -\mathbf{K}_{1, i i}^{-1} \mathbf{K}_{1, i b} \\
\mathbf{0} & \mathbf{0} & \mathbf{I}_{n_{b}} \\
\mathbf{0} & \boldsymbol{\Psi}_{2, i} & -\mathbf{K}_{2, i i}^{-1} \mathbf{K}_{2, i b} \\
\mathbf{0} & \mathbf{0} & \mathbf{I}_{n_{b}}
\end{array}\right]
$$

in which it can be seen that the validity of the condition Eq.(9) (previously highlighted in section 3.1) is ensured since $\mathbf{T}_{1, b}=\mathbf{T}_{2, b}$.

The Craig \& Bampton method is known to be applicable for dynamic model evaluation in a frequency range closely linked to the truncation of the component modes. The static attachment modes allow taking into account the interactions between the component and the modal contributions neglected by the modal truncation.

In practice this approach can be criticized since the number of Guyan condensed vectors $\boldsymbol{\Theta}_{1}$ and $\boldsymbol{\Theta}_{2}$ relies on the number of interface DoF $n_{b}$. The compactness of the Craig and Bampton-based reduced order model then decreases as the number of interface DoF $n_{b}$ increases.

\section{The proposed methodology}

This section presents the reduction method proposed in this article. This presentation is divided into three main key points: the primal assembly of the structural model of $\Sigma$ and the reduction of the model using component eigenmodes are presented first in (section 4.1). The main contribution of this article is the reduction of the interface and is explained in the section 4.2 .

\subsection{Primal direct coupling}

Conformity with the matrix Dirichlet equation (Eq.(4)) is imposed directly in the physical domain. Thus, unlike the component mode synthesis method (see section 3.1), the models treated by direct coupling methods are not reduced. The FETI method [6] provides typical examples of how direct coupling can be involved in reduction methods.

The direct primal coupling of two structures can be established starting from the junction equilibrium equation. In section 1 , the structural dynamic matrix equations of the uncoupled components $\Sigma_{1}$ and $\Sigma_{2}$ are given in Eq.(2) and Eq.(3), respectively. Once coupled, the junction equilibrium of the two structures can be written as follows:

$$
\left\{\begin{array}{l}
\mathbf{Z}_{1, b i} \mathbf{u}_{1, i}+\mathbf{Z}_{1, b b} \mathbf{u}_{1, b}=\mathbf{f}_{1, b}+\mathbf{g}_{1} \\
\mathbf{Z}_{2, b i} \mathbf{u}_{2, i}+\mathbf{Z}_{2, b b} \mathbf{u}_{2, b}=\mathbf{f}_{2, b}+\mathbf{g}_{2}
\end{array}\right.
$$


Vectors $\mathbf{g}_{1}$ and $\mathbf{g}_{2}$ denote the interaction forces that ensure the interactions of the two bodies. It is important to note that the interaction forces exist only on the contact interface. In order to guarantee conformity with the Dirichlet boundary conditions the assertion

$$
\mathbf{u}_{1, b}=\mathbf{u}_{2, b}=\mathbf{u}_{b}
$$

is set. Thus the force equilibrium at the interface is expressed as:

$$
\mathbf{Z}_{1, b i} \mathbf{u}_{1, i}+\left(\mathbf{Z}_{1, b b}+\mathbf{Z}_{2, b b}\right) \mathbf{u}_{b}+\mathbf{Z}_{2, b i} \mathbf{u}_{2, i}=\mathbf{f}_{1, b}+\mathbf{f}_{2, b}+\mathbf{g}_{1}+\mathbf{g}_{2}
$$

The coupling using a dual approach is based on the equilibrium of the interaction forces so that: $\mathbf{g}_{1}+\mathbf{g}_{2}=$ $\mathbf{0}$ [7]. In primal methods the computed displacement is exact by definition so the verification of the assertion $\mathbf{g}_{1}+\mathbf{g}_{2}=\mathbf{0}$ is always verified. Hence the dynamic stiffness of the model assembled can be written in the primal approach as:

$$
\mathbf{Z}(\omega)=\left[\begin{array}{ccc}
\mathbf{Z}_{1, i i} & \mathbf{Z}_{1, i b} & \mathbf{0} \\
\mathbf{Z}_{1, b i} & \mathbf{Z}_{1, b b}+\mathbf{Z}_{2, b b} & \mathbf{Z}_{2, b i} \\
\mathbf{0} & \mathbf{Z}_{2, i b} & \mathbf{Z}_{2, i i}
\end{array}\right] \text { with } \mathbf{u}=\left\{\begin{array}{c}
\mathbf{u}_{1, i} \\
\mathbf{u}_{b} \\
\mathbf{u}_{2, i}
\end{array}\right\}
$$

An interesting property of this assembled model Eq.(16) is that the symmetry of the assembled matrix $\mathbf{K}$ and $\mathbf{M}$ is kept. This allows using efficient numerical methods such as the Cholesky decomposition method to manage them.

To reduce the primal formulation of the coupled model Eq.(16) using component eigenmodes, it is possible to build a reduction basis $\mathbf{T}$ defined as follows:

$$
\mathbf{T}=\boldsymbol{\phi}_{l}=\left[\begin{array}{cc}
\boldsymbol{\phi}_{1, i} & \mathbf{0} \\
\boldsymbol{\phi}_{1, b} & \boldsymbol{\phi}_{2, b} \\
\mathbf{0} & \boldsymbol{\phi}_{2, i}
\end{array}\right]
$$

where $\boldsymbol{\phi}_{1}$ and $\boldsymbol{\phi}_{2}$ denote the truncated modal basis of the component $\Sigma_{1}$ and $\Sigma_{2}$.

Reference [19] explains that the use of free eigenmode components allows recovering the richness of the interface dynamics for low frequency behaviour only. Indeed, unlike the fixed-interface eigenmodes used in the Craig and Bampton method, the interface displacements of the free component eigenmodes are rather inconsistent, thus although the description of the component motion on their internal DoF is accurate, the interface displacements are described poorly. In practice this results in eigensolutions with non-null gaps $\Delta \mathbf{u}^{\Gamma}$, i.e. interpenetration or detachment.

Furthermore, Vermot Des Roches showed that the number of component eigenmodes required to enlarge the validity bandwidth of the reduced order model rapidly becomes large. Therefore improvement vectors must be used to increase the accuracy of the reduced model and verify the gap constraint $\Delta \mathbf{u}^{\Gamma}$ equation Eq.(4).

\subsection{Improvement vectors and interface reduction}

Coupling displacements are required to improve the accuracy of our reduced order model. The following criteria are used to build the vector bases:

- The coupling displacements must involve the "two-by-two" component and conform to the primal coupling condition (i.e. the gap $\Delta \mathbf{u}^{\Gamma}$ is null) in order to correct non-null gaps produced by the component eigenmodes. This approach was performed in [19, 4, 20, 14, 24].

- The contact interface must be reduced. Indeed, one of the main criticisms of the classical primal CMS method is that the description of the interface motion is performed using a nodal displacement basis that may lead to heavy reduced order models. Then a reduced basis of the interface displacements is sought. A review of the main interface reduction methods was written by Tran in [18]. 
In [19] system eigenmodes $\boldsymbol{\phi}_{1,2}$ were orthogonalized to the component modes and used as coupling displacements:

$$
\mathbf{T}=\left[\begin{array}{ll}
\mathbf{T}_{f} & \boldsymbol{\phi}_{1,2 \perp \mathbf{T}_{f}}
\end{array}\right]_{\text {orth }}
$$

In this way, it is possible to build a very accurate reduced order model with a small number of assembled system modes. However, in the present case it is assumed that knowledge of the system modes $\boldsymbol{\phi}_{1,2}$ is unavailable due to their computational cost.

Other more accessible interface reduction techniques using restrained eigenmodes were proposed in $[4,20,24,21]$. In the work of Aoyama and Yagawa [4] the improvement vectors are defined as the eigensolution of the problem of components assembled two-by-two. In the present case this corresponds to the assembled eigensolutions $\boldsymbol{\phi}_{1,2}$. In a recent publication of Jezequel and Garambois [25], the authors proposed and compared several DCMS (Double Component Modes Synthesis) for the Hellinger-Reissner (displacement-stress) formulation in structural dynamics. The DCMS methods studied in [25] used various combinations of fixed, free and branch modes for the modal analysis of two components tied to each other.

The principal contribution of this paper is to build a compact interface displacement basis from what is observed in the free eigenmodes of both components. To achieve this goal, a singular value decomposition of the component eigenmode interface restriction is used.

The desired basis is expected to recover all the interface motions observed. To ensure that all the interface motions will be well represented by the singular value decomposition it is important to normalize $\left[\begin{array}{ll}\boldsymbol{\phi}_{1, b} & \boldsymbol{\phi}_{2, b}\end{array}\right]$. Here, this normalization is performed for the interface mass matrix $\mathbf{M}_{1, b b}+\mathbf{M}_{2, b b}$ :

$$
\left[\begin{array}{ll}
\boldsymbol{\phi}_{1, b} & \boldsymbol{\phi}_{2, b}
\end{array}\right]^{\top}\left[\mathbf{M}_{1, b b}+\mathbf{M}_{2, b b}\right]\left[\begin{array}{ll}
\boldsymbol{\Phi}_{1, b} & \boldsymbol{\phi}_{2, b}
\end{array}\right]=\mathbf{I}_{n_{\boldsymbol{\Phi}_{1}+n_{\boldsymbol{\Phi}_{2}}}}
$$

The singular value decomposition allows the decomposition of the interface displacement matrix $\left[\begin{array}{ll}\boldsymbol{\phi}_{1, b} & \boldsymbol{\phi}_{2, b}\end{array}\right]$ into left singular vectors $\Upsilon$, a singular value matrix $\boldsymbol{\Lambda}=\operatorname{diag}\left(\lambda_{1}, \ldots, \lambda_{n}\right)$ and right singular vectors $\mathbf{V}$ as follows:

$$
\left[\begin{array}{ll}
\boldsymbol{\phi}_{1, b} & \boldsymbol{\phi}_{2, b}
\end{array}\right]=\mathbf{\Upsilon} \boldsymbol{\Lambda} \mathbf{V}^{\top}
$$

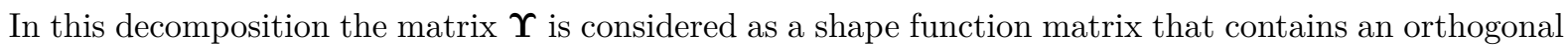
basis of interface displacements sorted as a function of their associated singular values $\lambda$. This approach to building a basis $\Upsilon$ for interface reduction is the main contribution of this work.

Thus, once the singular value $\lambda$ associated with the vectors of the basis $\Upsilon$ is known, it is possible to build a basis capable of generating all the interface displacements observed in the component modes. The vectors associated with the biggest singular values $\lambda$ are the most significant for optimising the recovery of the interface displacement observed. This means that the size of the basis $\Upsilon \mathbf{c}$ can be optimized by truncation with respect to the associated singular value $\lambda$.

The displacements in $\boldsymbol{\Upsilon}$ are common to both components so that we define $\boldsymbol{\Theta}_{\boldsymbol{\Upsilon}}$ as the uplift of the component interior DoF around the interface displacement of the basis $\boldsymbol{\Upsilon}$. Since all the interface displacements $\boldsymbol{\Upsilon}$ can be recovered by the component eigenmodes vectors $\boldsymbol{\phi}_{l}$ (Eq.(17)), it is unnecessary to include $\boldsymbol{\Upsilon}$ in $\Theta_{\Upsilon}$ :

$$
\boldsymbol{\Theta}_{\Upsilon}=\left[\begin{array}{cc}
-\mathbf{Z}_{1, i i}^{-1} \mathbf{Z}_{1, i b} \mathbf{\Upsilon} & \mathbf{0} \\
\mathbf{0} & \mathbf{0} \\
\mathbf{0} & -\mathbf{Z}_{2, i i}^{-1} \mathbf{Z}_{2, i b} \mathbf{\Upsilon}
\end{array}\right]
$$

In Guyan's condensation method, Eq.(12) is computed with $\omega=0$. In the present work, the Eq.(21) is computed with different values of $\omega$ in order to improve the reduction basis. Naturally, these operations are time consuming since they require the inversion of $\mathbf{Z}_{1, i i}$ and $\mathbf{Z}_{2, i i}$. Moreover, the bigger the reduction basis is, the lower the reduction efficiency. In this work we arbitrarily choose to calculate only the coupling 
displacement for $\omega_{\min }, \omega_{\max }$ and $\omega_{\operatorname{mid}}=\frac{1}{2}\left(\omega_{\max }+\omega_{\min }\right)$ of the waveband studied $\Delta \omega$. This leads to the following reduction:

$$
\mathbf{T}=\left[\begin{array}{llll}
\boldsymbol{\Phi}_{l} & \boldsymbol{\Theta}_{\Upsilon}\left(\omega_{\text {min }}\right) & \boldsymbol{\Theta}_{\Upsilon}\left(\omega_{\text {mid }}\right) & \boldsymbol{\Theta}_{\Upsilon}\left(\omega_{\max }\right)
\end{array}\right]
$$

The reasoning behind the choice of the circular frequencies $\omega$ is that using frequencies regularly spread over the bandwidth of interest $\Delta f$ will help recovering the dynamics of the system over $\Delta f$. Indeed, the contributions of the modes whose natural frequencies are distant from the frequencies $\omega$ tend to be neglected. The choice of the circular frequencies is a strategic choice that should be made with regards to the case study considered and the goal targeted.

This leads to the reduction basis $\mathbf{T}$ of size $n_{T}=n_{\phi_{1}}+n_{\phi_{2}}+6 n_{\Upsilon}$. The reduction basis $\mathbf{T}$ (Eq.(22)) is orthogonalised with regards to the assembled mass matrix $\mathbf{M}$ to improve the numerical conditioning of the reduced order model produced by the reduction methodology proposed, thus:

$$
\mathbf{T}^{\top} \mathbf{M T}=\mathbf{I}_{n_{T} \times n_{T}}
$$

The reduction methodology proposed in this section enables building a model whose accuracy and size can be tuned thanks to the singular values. The interface modes obtained using the SVD allow describing the interface motions observed in the component eigenmodes with the desired precision.

\subsection{Reduced order model update}

The reduced order model of the hub-blade assembly (Fig.6b) changes considerably in the framework of iterative blade design. Although the finite element model of the blade (Fig.6a) is frequently redesigned, the hub remains unchanged. Thus, instead of entirely rebuilding the reduced order model at each design iteration, it is interesting to identify which term corresponding to the blade has to be updated. In this section, the system considered has only two components (the hub and the blade); however the results presented can be extended to an assembly with more components.

When regrouping the component free eigenmodes Eq.(17) and the improvement vectors Eq.(21) in the reduction basis Eq.(22) $\mathbf{T}$ can be expressed as follows:

$$
\mathbf{T}=\left[\begin{array}{cccc}
\boldsymbol{\Phi}_{1 i} & \mathbf{0} & \boldsymbol{\Theta}_{1, \Upsilon} & \mathbf{0} \\
\boldsymbol{\Phi}_{1 b} & \boldsymbol{\Phi}_{2 b} & \mathbf{0} & \mathbf{0} \\
\mathbf{0} & \boldsymbol{\Phi}_{2 i} & \mathbf{0} & \boldsymbol{\Theta}_{2, \Upsilon}
\end{array}\right]
$$

The reduction of the assembled matrix Eq.(16) using the reduction basis Eq.(24) leads to a reduced matrix $\tilde{\mathbf{Z}}$ with the following symmetrical topology:

$$
\tilde{\mathbf{Z}}=\left[\begin{array}{cccc}
\tilde{\mathbf{Z}}_{11} & \tilde{\mathbf{Z}}_{12} & \tilde{\mathbf{Z}}_{13} & \tilde{\mathbf{Z}}_{14} \\
\text { sym. } & \tilde{\mathbf{Z}}_{22} & \tilde{\mathbf{Z}}_{23} & \tilde{\mathbf{Z}}_{24} \\
\text { sym. } & \text { sym. } & \tilde{\mathbf{Z}}_{33} & \mathbf{0} \\
\text { sym. } & \text { sym. } & \text { sym. } & \tilde{\mathbf{Z}}_{44}
\end{array}\right]
$$

The terms of the reduced matrix Eq.(25) are given below:

$$
\begin{array}{ll}
\tilde{\mathbf{Z}}_{11}=\left(-\omega^{2} \mathbf{I}_{n_{\boldsymbol{\phi}_{1}}}+\boldsymbol{\Lambda}_{1}\right)+\boldsymbol{\phi}_{1 b}^{\top} \mathbf{Z}_{2 b b} \boldsymbol{\phi}_{1 b} & \tilde{\mathbf{Z}}_{12}=\left(\boldsymbol{\phi}_{1 i}^{\top} \mathbf{Z}_{1, i b}+\boldsymbol{\phi}_{1 b}^{\top}\left(\mathbf{Z}_{1, b}+\mathbf{Z}_{2, b}\right)+\boldsymbol{\phi}_{1 b}^{\top} \mathbf{Z}_{2, i b}\right) \boldsymbol{\phi}_{2, b} \\
\tilde{\mathbf{Z}}_{22}=\left(-\omega^{2} \mathbf{I}_{n_{\boldsymbol{\Phi}_{2}}}+\boldsymbol{\Lambda}_{2}\right)+\boldsymbol{\phi}_{2 b}^{\top} \mathbf{Z}_{1 b b} \boldsymbol{\Phi}_{2 b} & \tilde{\mathbf{Z}}_{13}=\left(\boldsymbol{\phi}_{1 b}^{\top} \mathbf{Z}_{1, b i}+\boldsymbol{\phi}_{1 i}^{\top} \mathbf{Z}_{1, i i}\right) \boldsymbol{\Theta}_{1, \Upsilon} \\
\tilde{\mathbf{Z}}_{33}=\boldsymbol{\Theta}_{1, \Upsilon}^{\top} \mathbf{Z}_{1, i i} \boldsymbol{\Theta}_{1, \Upsilon} & \tilde{\mathbf{Z}}_{14}=\boldsymbol{\phi}_{1 b}^{\top} \mathbf{Z}_{2 b i} \boldsymbol{\Theta}_{2, \Upsilon} \\
\tilde{\mathbf{Z}}_{44}=\boldsymbol{\Theta}_{2, \Upsilon}^{\top} \mathbf{Z}_{2, i i} \boldsymbol{\Theta}_{2, \Upsilon} & \tilde{\mathbf{Z}}_{23}=\boldsymbol{\phi}_{2 b}^{\top} \mathbf{Z}_{1, b i} \boldsymbol{\Theta}_{1, \Upsilon} \\
& \tilde{\mathbf{Z}}_{24}=\left(\boldsymbol{\phi}_{2 b}^{\top} \mathbf{Z}_{2, b i}+\boldsymbol{\phi}_{2 i}^{\top} \mathbf{Z}_{2, i i}\right) \boldsymbol{\Theta}_{2, \Upsilon}
\end{array}
$$


The modification of one component $\left(\Sigma_{1}\right.$ or $\left.\Sigma_{2}\right)$ affects all the terms of $\tilde{\mathbf{Z}}$. For instance, let us consider the modification of the component $\Sigma_{2}$, which impacts $\boldsymbol{\phi}_{2}, \boldsymbol{\Theta}_{2, \Upsilon}$ and $\boldsymbol{\Theta}_{1, \Upsilon}$. In this case, all the terms of $\tilde{\mathbf{Z}}$ have to be updated.

Nevertheless, it is possible to consider that the modification of $\Sigma_{2}$ does not significantly impact $\mathbf{\Upsilon}$. This can be measured by verifying that the interface basis $\boldsymbol{\Upsilon}$ allows accurately recovering the displacements expressed by the modified term $\boldsymbol{\phi}_{2 b}$. In practice, this can be performed by checking that the norm of the residue $\mathbf{r}$ associated with each vector $\boldsymbol{\phi}_{2 b}$ is small:

$$
\mathbf{r}=\left(\mathbf{I}-\Upsilon \boldsymbol{\Upsilon}^{\top}\right) \boldsymbol{\phi}_{2 b}
$$

Under this assumption, the terms $\tilde{\mathbf{Z}}_{13}$ and $\tilde{\mathbf{Z}}_{33}$ are no longer dependent on the modifications applied to $\Sigma_{2}$. Consequently, the modification of $\Sigma_{2}$ leads only to the re-computation of 7 of the 16 terms of $\tilde{\mathbf{Z}}_{\text {: }} \tilde{\mathbf{Z}}_{11}$, $\tilde{\mathbf{Z}}_{22}, \tilde{\mathbf{Z}}_{44}, \tilde{\mathbf{Z}}_{12}, \tilde{\mathbf{Z}}_{14}, \tilde{\mathbf{Z}}_{23}$ and $\tilde{\mathbf{Z}}_{24}$.

Starting from a reduced model $\tilde{\mathbf{Z}}$, updating the reduced matrix due to the modification of $\Sigma_{2}$ is managed as follows:

1. parallel re-computation of $\boldsymbol{\phi}_{2}$ and $\boldsymbol{\Theta}_{2, \Upsilon}$.

2. orthogonalisation $\boldsymbol{\Theta}_{2, \Upsilon}$ to $\boldsymbol{\phi}_{2 i}$.

3. parallel re-computation of $\tilde{\mathbf{Z}}_{11}, \tilde{\mathbf{Z}}_{22}, \tilde{\mathbf{Z}}_{44}, \tilde{\mathbf{Z}}_{12}, \tilde{\mathbf{Z}}_{14}, \tilde{\mathbf{Z}}_{23}$ and $\tilde{\mathbf{Z}}_{24}$.

4. reassembly of $\tilde{\mathbf{Z}}$ using the term previously re-computed.

\section{Application}

The application of the methodology proposed is first described and discussed through an academic case study with few DOF. Secondly, the reduction of a real industrial case study is performed and its results are compared with those obtained with the Craig and Bampton method.

The mass modal assurance criterion (mass-MAC) is a reference method [26] that allows comparing the orthogonality of modes with respect to the mass matrix of a given finite element model. In the present case, the accuracy of the two methods is compared using the mass-MAC computed in the nodal (HFM) space.

\subsection{Academic case study with high interface dynamics}

Let us consider the finite element model (7860 DoF) whose mesh is presented in Fig.2. The model is composed of two parts, $\Sigma_{1}$ and $\Sigma_{2}$, and the system assembled is studied over the frequency range $\Delta f=[0,3000]$ Hz. The contact interface $\Gamma$ has 321 DoF. Over $\Delta f$ the components have $n_{\boldsymbol{\Phi}_{1}}=29$ and $n_{\boldsymbol{\Phi}_{2}}=22$ modes.

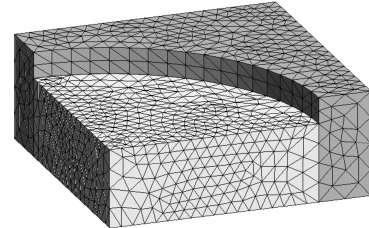

(a)

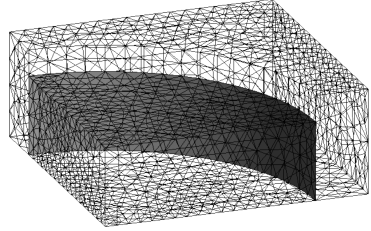

(b)

Figure 2: The case study is composed of two parts: $\Sigma_{1}$ (in dark gray) with 3747 DoF and $\Sigma_{2}$ (in light gray) with 3933 DoF. (a) Mesh of the assembled case study; (b) Contact interface $\Gamma$ between $\Sigma_{1}$ and $\Sigma_{2}$ 
The SVD-basis $\Upsilon \Upsilon$ is built from the 51 component eigenmodes found in the frequency range $[0 ; 3000]$ $\mathrm{Hz}$, once normalized (Eq.(19)), the singular value decomposition of the interface displacements provide the basis $\Upsilon$ (Eq.(20)). In Fig.3, the logarithmic evolution of the singular values $\lambda$ showing a truncation at the $45^{\text {th }}$ vector of $\Upsilon$ appears logical.

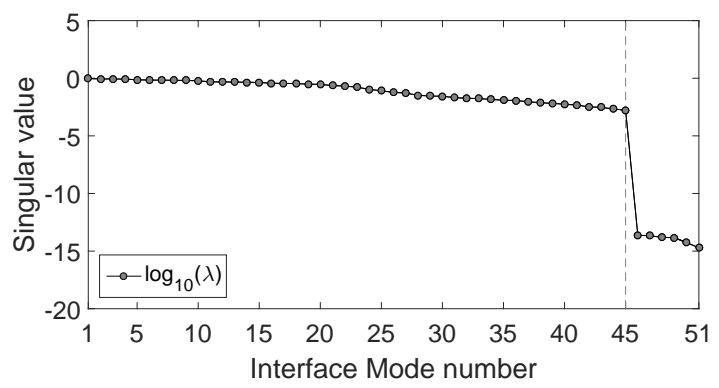

Figure 3: Singular values associated with the displacement component of the basis $\mathbf{\Upsilon}$.

The MAC average is evaluated for several truncations to study the impact of the $\Upsilon$ truncation on the quality of the results. In Fig.4 it can be seen that, in practice, the impact of the truncation is significant around 45 vectors. Indeed, using more than 45 interface motions does not significantly improve the quality of the results.

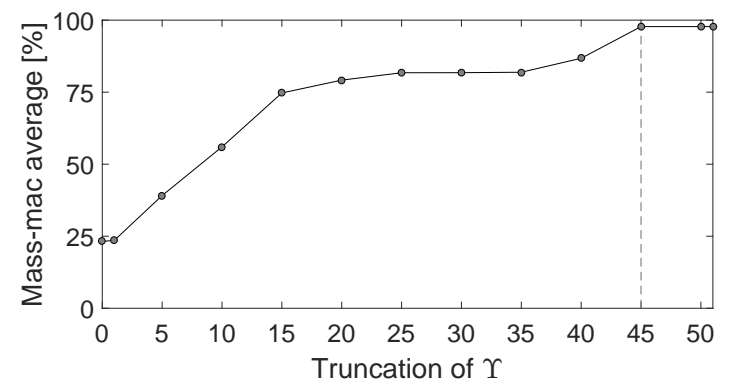

Figure 4: Mass-MAC average evaluated for a reduced order model constructed using different truncations of $\Upsilon$

In section 4.2 the choice of using several circular frequencies was justified since it leads to better recovery of the assembled eigenmodes spread on the frequency band of interest. The Fig. 5 gives the mass-MAC results obtained using different circular frequencies $\omega$.

As can be seen in Fig.5, the improvement vectors computed for 1500 and 3000 Hertz improves the solutions of the highest frequencies. Let us conclude this first study by comparing the results obtained using the method proposed and the Craig \& Bampton method. 


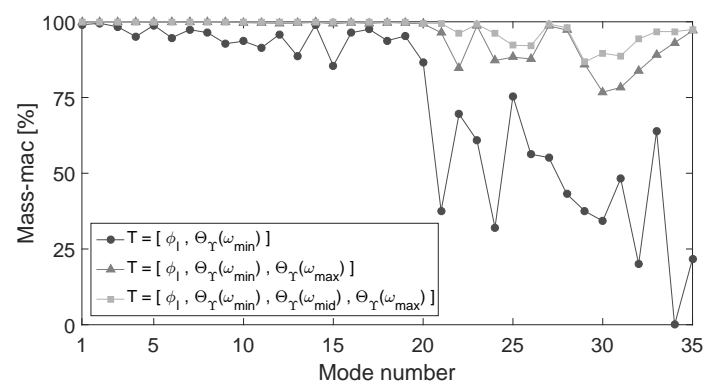

Figure 5: Comparison of the mass-MAC obtained with different reduced order models built with varying dynamic condensations of the components and an interface displacement basis $\Upsilon$ truncated at its $45^{\text {th }}$ element.

\begin{tabular}{llll} 
Method & $\begin{array}{l}\text { Craig and } \\
\text { method }\end{array}$ & Bampton & $\begin{array}{l}\text { Methodology proposed us- } \\
\text { ing the first } 45^{\text {th }} \text { vectors of } \\
\Upsilon\end{array}$ \\
\hline Basis size & 393 & 321 \\
\hline MAC average & $98.59 \%$ & $97.76 \%$ \\
\hline Frequency error average & $0.35 \%$ & $0.52 \%$
\end{tabular}

For this specific application it can be seen that the Craig \& Bampton method provides better results than the method proposed, although its associated reduced order model is less compact.

\subsection{Industrial case study}

To validate our premise we apply the methodology described previously to the blade of an open rotor aircraft engine tied to a hub at its root. The assembly (Fig.6b) contains 189888 DoF and is studied over a frequency bandwidth $\Delta f$ between 0 and $2000 \mathrm{~Hz}$. Over this range, the system assembled has 53 flexible eigenmodes that should all be recovered by the reduced order model proposed. The behaviour of both parts is assumed to be linear.

To establish a close link between the theory previously introduced and its concrete application the hub will be denoted $\Sigma_{1}$ while $\Sigma_{2}$ refers to the blade model in the next section.

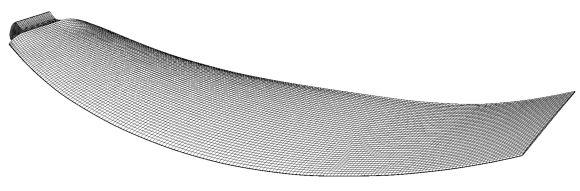

(a)

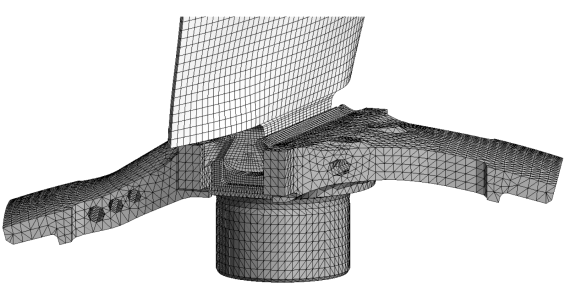

(b)

Figure 6: The mesh of the blade $\Sigma_{2}$ is composed of 39724 nodes $\left(n_{2}=119172 \mathrm{DoF}\right)$ while 24370 nodes $\left(n_{1}=70716\right.$ DoF $)$ are used for the hub mesh $\Sigma_{1}$. (a) Mesh of the whole blade; (b) The hub $\Sigma_{1}$ is assembled with the blade $\Sigma_{2}$. 
The method proposed has been developed in the framework of the iterative design of the blade. The blade geometry is modified frequently whereas the hub remains unchanged. In the present application however, only one blade design is tested, thus the ideas presented in 4.3 are not exploited.

\subsubsection{Application of the Craig and Bampton method}

The component fixed-interface eigenmodes are truncated using Rubin's criterion over the frequency range $\left[0,1.5 \times f_{\max }\right]=[0,3000]$ Hertz, which corresponds to 67 reduction vectors.

The size of the reduced order model obtained using the fixed-interface interface mode and the component static condensation is $1147 \times 1147$. This reduced order model has an average relative frequency error of $0.26 \%$ (see Fig.9) while its average MAC reaches more than $94 \%$ (see Fig.10a).

As the first 6 eigensolutions are identified as rigid body motions, their associated eigenvalues vanish, thus the associated relative frequency error tends to infinity. This explains why these 6 relative frequency errors are not plotted in the Fig.9.

As can be seen in Fig.10a, some eigensolutions are badly approximated with a MAC below $60 \%$. A reference reduced order model was built that will now be subjected to the method proposed, in order to compare its results with those of the Craig and Bampton method.

\subsubsection{Application of the method proposed}

The first step of the method proposed is the computation of the component free eigenmodes. The selection of the component eigenmodes is a key point of the modal truncation that can be treated in various ways. In the present case we decided to use only the component eigenmodes over the frequency range $[0,2000] \mathrm{Hz}$ which gives $n_{\boldsymbol{\Phi}_{1}}=9$ modes for the hub and $n_{\boldsymbol{\Phi}_{2}}=56$ modes for the blade.

The singular value decomposition of the component interface displacement leads to the truncated basis $\Upsilon$ of the interface motion which has $65\left(n_{\boldsymbol{\Phi}_{1}}+n_{\boldsymbol{\Phi}_{2}}\right)$ modes. From the singular values $\lambda_{i}$ associated with these motions $v_{i}$ (Fig.7) it can be seen that the interface displacement of the component eigenmodes can be brought about using a very restricted SVD-basis:

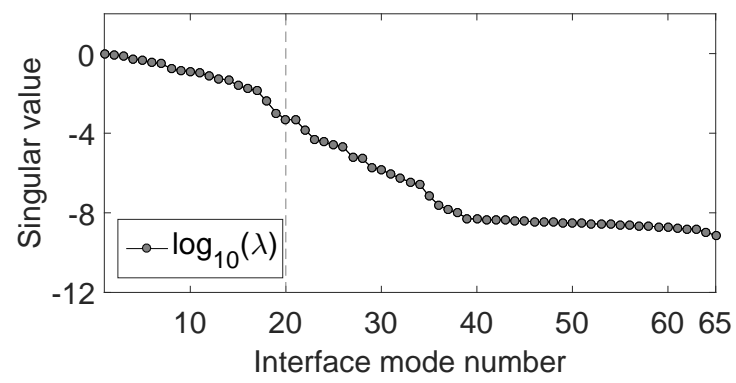

Figure 7: Singular values associated with the component of the basis $\Upsilon$.

The fall of the singular value is slower than that obtained in the first case study Fig.3. In this case the threshold was set by considering one thousandth of the first eigenvalue. Using this criterion, the description of the interface motion over [0,2000] Hz is assumed using 20 DoF while a nodal description of the interface motion would have required 1080 DoF. 
Several interface displacements of $\mathbf{\Upsilon}$ are given as examples in Fig.8. The first 12 displacements correspond to rigid modes; indeed, the contact interface is split into two parts, each having 6 rigid modes.

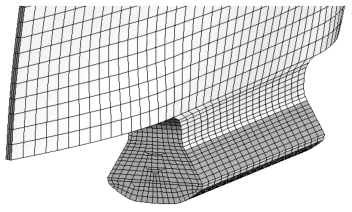

(a)

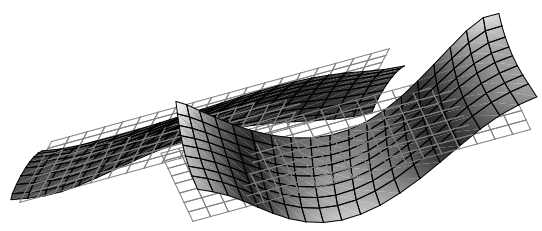

(c)

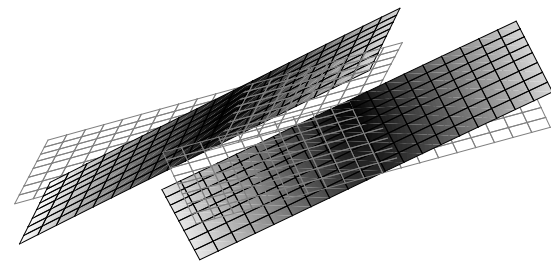

(b)

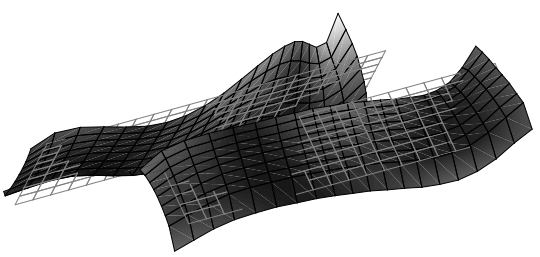

(d)

Figure 8: Example of contact interface displacements obtained from the singular value decomposition of the component eigenmode interface restriction. (a) Contact interface between the blade and the hub; (b) Interface displacement $v_{5}, \lambda_{5}=5.36$; (c) Interface displacement $v_{15}, \lambda_{15}=1.48 \times 10^{-2}$; (d) Interface displacement $v_{22}, \lambda_{22}=4.29 \times 10^{-4}$;

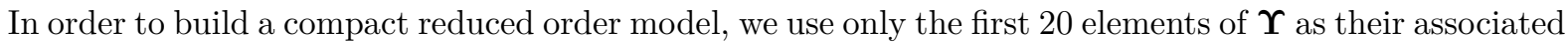
singular values are much higher than the others (see Fig.7). The computation of the improvement vectors is performed around the truncated $\boldsymbol{\Upsilon}$ and for 0,1000 and $2000 \mathrm{~Hz}$. Thus, once built, the reduced order model has only $n=185 \mathrm{DoF}$ (while the high fidelity model has $189888 \mathrm{DoF}$ ), indeed $n=n_{\boldsymbol{\Phi}_{1}}+n_{\boldsymbol{\Phi}_{2}}+6 \times 20$.

As can be seen in Fig.9, the reduced order model built provides good results in terms of MAC and relative frequency error. Indeed the averages of the relative frequency (0.18\%, see Fig.9) and the MAC (99.28, see Fig.10b) are better than those obtained using the Craig and Bampton method.

As with the previous case, the relative errors associated with the first six eigenfrequencies are not plotted in Fig.9.

It is interesting to compare the subspace generated by the Craig \& Bampton method and the method proposed. Let us compare the orthogonality between the proposed reduction basis $\mathbf{T}$ (that has $n$ vectors) and the Craig and Bampton reduction basis. Considering the same DoF set, the vectors of the reduction basis proposed can be split into an orthogonal part $\mathbf{T}_{\perp}$ and a part parallel $\mathbf{T}_{/ /}$to the Craig and Bampton reduction basis so that:

$$
\mathbf{T}=\mathbf{T}_{\perp}+\mathbf{T}_{/ /}
$$

The orthogonal part $\mathbf{T}_{\perp}$ is computed using the Gram-Schmidt base procedure. We observe that the vectors of the proposed methods are relatively parallel since we found that:

$$
\frac{100}{n} \times \sum_{i=1}^{n} \frac{\|\mathbf{T} / /\|^{2}}{\|\mathbf{T}\|^{2}} \geq 99.9
$$

In conclusion, we can say that the subspace generated by the Craig and Bampton method and the method proposed overlap. 

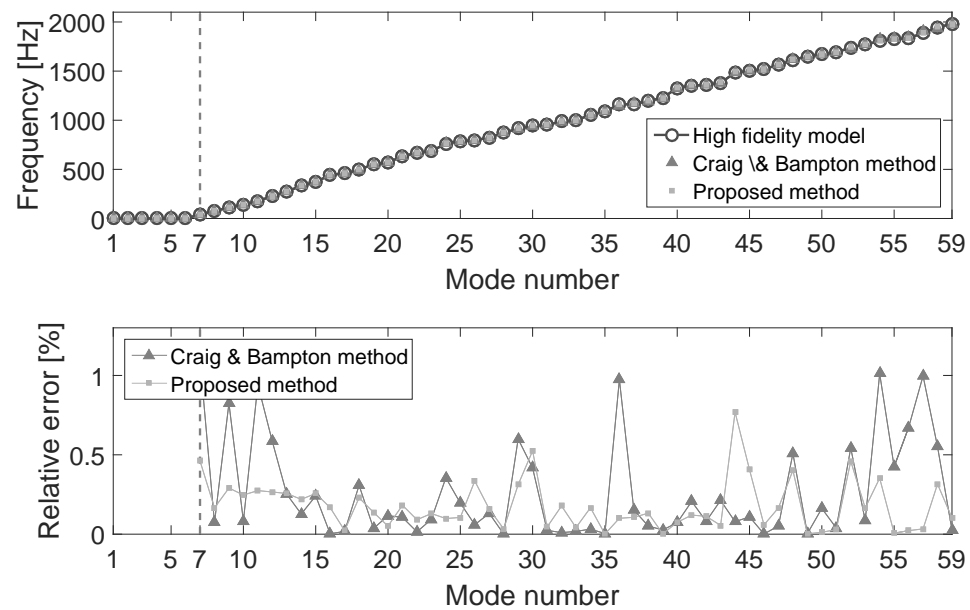

Figure 9: Comparison between the model eigenfrequencies assembled with Matlab and the proposed reduced order model.

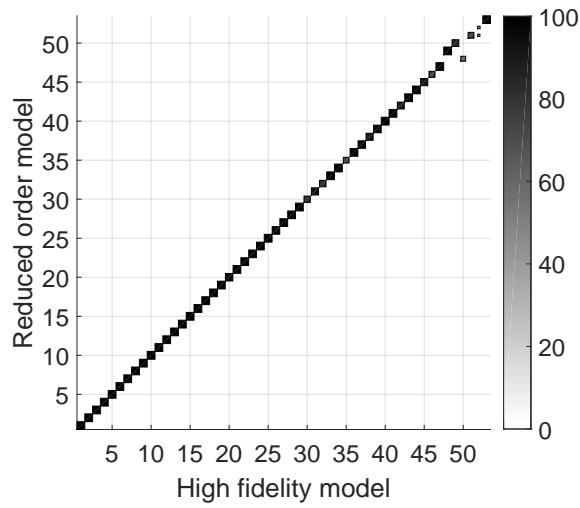

(a)

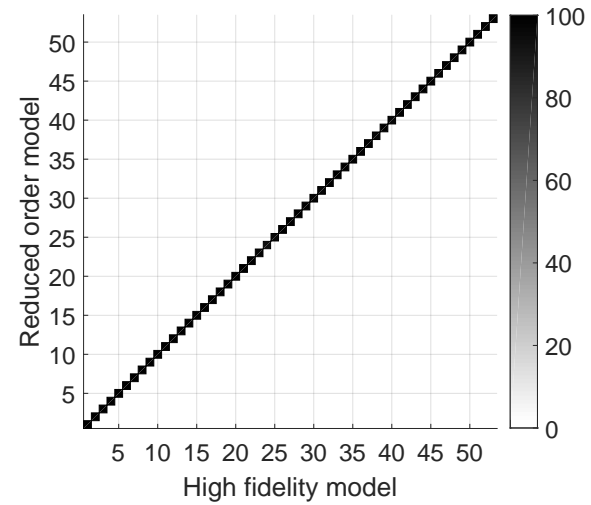

(b)

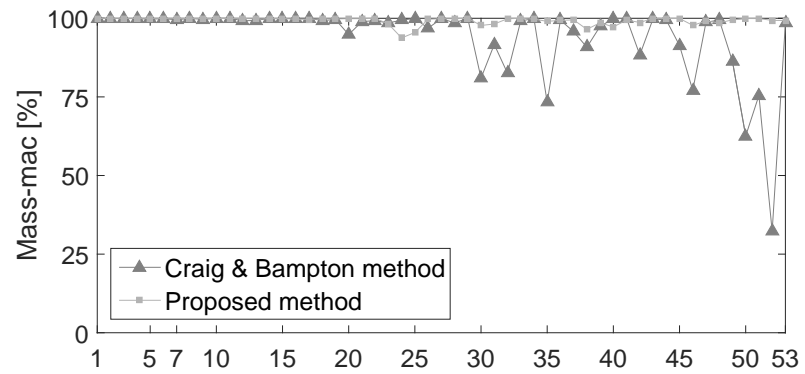

(c)

Figure 10: Mass-MAC average of the Craig \& Bampton method and the method proposed. (a) Craig \& Bampton method: number of DoF: 1147, MAC average: 94.37\% (min.: 32.30\%); (b) Proposed method: number of DoF: 185, MAC average: 99.28\% (min.: 93.75\%); (c) Comparison of the mass-MAC obtained with the Craig \& Bampton method and the proposed method; 


\subsection{Summary of results}

The results of the previous experiment are summarized in the following table

\begin{tabular}{llll} 
Method & $\begin{array}{l}\text { Craig and } \\
\text { method }\end{array}$ & Bampton & $\begin{array}{l}\text { Proposed methodology } \\
\text { using the first } 20^{t h} \text { vectors } \\
\text { of } \mathbf{\Upsilon}\end{array}$ \\
\hline Basis size & 1147 & 185 \\
\hline MAC average & $94.37 \%$ & $99.28 \%$ \\
\hline Frequency error average & $0.26 \%$ & $0.18 \%$
\end{tabular}

\section{Conclusion}

In this paper we proposed a modal analysis oriented reduction methodology. The method proposed is a powerful tool in structural dynamics for building reduced order models of assembled structures with free component eigenmodes and without any dynamic simulation of the complete structure. Good compactness can be provided by the interface reduction method proposed in section 4, which is the central contribution of this work. In section 4.1 we showed that the matrix of the reduced order model is open to tuning. Indeed, model tuning can be considered through the direct modification of the component eigenvalues that appear in the terms $\tilde{\mathbf{Z}}_{11}$ and $\tilde{\mathbf{Z}}_{22}$ highlighted in section 4.3.

The main approach set out in this article, i.e. interface reduction using the SVD of the component freefree eigenmodes, is efficient and has several advantages. It is a simple and powerful technique that allows recovering most of the interface motions of the system assembled. Moreover, the singular values can be used to filter the interface displacement and then to manage the compactness and the precision of a reduced order model. Contrary to the Craig and Bampton method, the number of interface DoFs is no longer linked to its mesh but to its dynamics. The method's efficiency increases as the interface dynamics decrease, which can be seen when comparing the compactness of the reduced order model obtained for the different case studies.

When applied it could be seen clearly that the method proposed enabled us to obtain an accurate and compact reduction basis in terms of MAC (average: 99.28\%, see Fig.10b) and relative frequency error (average: $0.18 \%$, see Fig.9). The number of degrees of freedom fell drastically from 189888 to 185 with our reduction method while the Craig and Bampton method required 1147 DoF and provided less accurate results: MAC average $94.37 \%$, relative frequency error $0.26 \%$.

The efficiency of the method proposed is better for case studies with low interface dynamics. This was visible in particular for the first case study, for which the method proposed was about equivalent to the Craig \& Bampton method in terms of accuracy and compactness.

In conclusion, the article presented a reduction method inspired from the dynamic substructuring technique and characterized by the three following features:

- the interface is reduced using a basis $\Upsilon \Upsilon$ built from component eigenmodes;

- the methodâÁŹs accuracy is roughly equivalent to that of the Craig and Bampton method but produces smaller reduced order models thanks to the interface reduction;

- the reduction is intended for modal analysis in the context of redesign. 


\section{Acknowledgments}

This research work was carried out under the leadership of the Technological Research Institute SystemX and therefore benefited from public funds within the scope of the French Program «Investissements d'Avenir ». In addition, we are grateful to SNECMA and the Laboratoire QUARTZ for their implication and cooperation in the current work.

[1] B. Besselink, U. Tabak, A. Lutowska, N. van de Wouw, H. Nijmeijer, D.J. Rixen, M.E. Hochstenbach, and W.H.A. Schilders, A comparison of model reduction techniques from structural dynamics, numerical mathematics and systems and control, Journal of Sound and Vibration, 332 (2013) 4403-4422.

[2] U. Hetmaniuk, R. Tezaur, and C. Farhat, Review and assessment of interpolatory model order reduction methods for frequency response structural dynamics and acoustics problems, International Journal for Numerical Methods in Engineering, 90 (2011) 1636-1662.

[3] J.V. Aguado, A. Huerta, F. Chinesta, and E. Cueto, Real-time monitoring of thermal processes by reduced order modeling, International Journal for Numerical Methods in Engineering, 102 (2015) 991-1017.

[4] Y. Aoyama and G. Yagawa, Component mode synthesis for large-scale structural eigenanalysis, Computers 83 Structures, 79 (2001) 605-615.

[5] R. Shioya and G. Yagawa, Parallel finite elements of ten-million dofs based on domain decomposition method, WCCM IV, Computational Mechanics-New Trends and Applications, 11 (1998) 1-12.

[6] C. Farhat, J. Li, and P. Avery, A FETI-DP method for the parallel iterative solution of indefinite and complex-valued solid and shell vibration problems, International Journal for Numerical Methods in Engineering, 63 (2005) 398-427.

[7] D. De Klerk, D.J. Rixen, and S.N. Voormeeren, General Framework for Dynamic Substructuring: History, Review and Classification of Techniques, AIAA Journal, 46 (2008) 1169-1181.

[8] A.L. Hale and L. Meirovitch, A general substructure synthesis method for the dynamic simulation of complex structures, Journal of Sound and Vibration, 69 (1980) 309-326.

[9] M.F. Kaplan, Implementation of automated multilevel substructuring for frequency response analysis of structures, PhD Thesis, University of Texas at Austin, USA, 2001.

[10] W. Gao, X.S. Li, C. Yang, and Z. Bai, An Implementation and Evaluation of the AMLS Method for Sparse Eigenvalue Problems, ACM Transactions on Mathematical Software (TOMS), 5 (2008) 1-27.

[11] R.H. MacNeal, A hybrid method of component mode synthesis, Computers Es Structures, 1 (1971) 581-601.

[12] D.J. Rixen, A dual Craig-Bampton method for dynamic substructuring. Journal of Computational and Applied Mathematics, 168 (2004) 383-391.

[13] A. Shanmugam and C. Padmanabhan. A fixed-free interface component mode synthesis method for rotordynamic analysis, Journal of Sound and Vibration, 297 (2006) 664-679.

[14] N. Bouhaddi and J.P. Lombard, Improved free-interface substructures representation method, Computers and Structures, 77 (2000) 269-283.

[15] Ch. U. A. Bucher, A modal synthesis method employing physical coordinates, free component modes, and residual flexibilities, Computers \& Structures, 22 (1986) 559-564.

[16] C. Farhat and M. Géradin, On a component mode synthesis method and its application to incompatible substructures, Computers \& Structures, 51 (1994) 459-473.

[17] D.R. Martinez, A.K. Miller, and T.G. Carne, Combined experimental/analytical modeling of shell/payload structures, Technical report, Sandia National Labs., Albuquerque, USA, 1985.

[18] D.M. Tran, Component mode synthesis methods using partial interface modes: Application to tuned and mistuned structures with cyclic symmetry, Computers \& Structures, 87 (2009) 1141-1153.

[19] G. Vermot des Roches, Frequency and time simulation of squeal instabilities Application to the design of industrial automotive brakes, PhD Thesis, Ecole Centrale Paris, France, 2011.

[20] K. Brahmi, N. Bouhaddi, and R. Fillod, Reduction of Junction Degrees of Freedom in Certain Methods of Dynamic Substructure Synthesis, Proceedings-SPIE The International Society for Optical Engineering. SPIE International Society for Optical, (1995) $1763-1769$.

[21] D.M. Tran, Component mode synthesis methods using interface modes: Application to structures with cyclic symmetry, Computers \& Structures, 87 (2001) 1141-1153.

[22] O. Dorival, P. Rouch, and O. Allix, A substructured version of the variational theory of complex rays dedicated to the calculation of assemblies with dissipative joints in the medium-frequency range, Engineering Computations, 23 (2006) $729-748$.

[23] F. Tisseur and K. Meerbergen, The quadratic eigenvalue problem, SIAM Review, 43 (2001) 235-286.

[24] H. Hassis, Proposition of a New Approach for the Substructure Method. Journal of Sound and Vibration, 232 (2000) 659-668.

[25] P. Garambois, S. Besset, and L. Jézéquel, Various double component mode synthesis and sub-structuring methods for dynamic mixed FEM, European Journal of Mechanics, A/Solids, 53 (2015) 196-219.

[26] R.J. Allemang, The Modal Assurance Criterion - Twenty Years of Use and Abuse, Sound and Vibration, 37 (2003) 14-20. 